\title{
Capture of interplanetary bodies in geocentric orbits and early lunar evolution
}

\author{
Malapaka ShivakumaR ${ }^{1,2, *}$ and N Bhandari ${ }^{1,3, * *}$ \\ ${ }^{1}$ Physical Research Laboratory, Ahmedabad 380 009, India. \\ ${ }^{2}$ University of Hyderabad, Hyderabad 500 046, India. \\ ${ }^{3}$ Basic Sciences Research Institute, Navrangpura, Ahmedabad 380 009, India. \\ *e-mail: shiva_astro@yahoo.co.in \\ **e-mail: bhandari@prl.ernet.in
}

During the accretion of planets such as Earth, which are formed by collisional accretion of planetesimals, the probability of capture of interplanetary bodies in planetocentric orbits is calculated following the approach of Hills (1973) and the $n$-body simulation, using simplectic integration method. The simulation, taking an input mass equal to about $50 \%$ of the present mass of the inner planets, distributed over a large number of planetoids, starting at $4 \mathrm{My}$ after the formation of solar system, yielded four inner planets within a period of $30 \mathrm{My}$. None of these seed bodies, out of which the planets formed, remained at this time and almost $40 \%$ mass was transferred beyond $100 \mathrm{AU}$. Based on these calculations, we conclude that $\sim 1.4$ times the mass of the present inner planets was needed to accumulate them. The probability of capture of planetoids in geocentric orbits is found to be negligible. The result emphasizes the computational difficulty in 'probability of capture' of planetesimals around the Earth before the giant impact. This conclusion, however, is in contradiction to the recent observations of asteroids being frequently captured in transient orbits around the Earth, even when the current population of such interplanetary bodies is smaller by several orders of magnitude compared to the planetary accumulation era.

\section{Introduction}

The formation of various bodies in the solar system, starting from the solar nebula, is currently understood in terms of the standard model of planet formation given by Safronov (1969), Wetherill (1980, 1986) and Wetherill and Stewart (1993). The astronomical observations of proto-planetary disks, chemical, isotopic and chronological studies of meteorites and other extra terrestrial samples support this model. Although the formation of inner rocky planets, outer giant gaseous planets and the outermost icy planets, asteroids, Kuiper belt objects and comets are generally understood within the frame work of this model, the formation of the Moon requires an ad hoc, rare event of collision of a giant object
$\left(M=0.1 M_{E}\right)$ with the infant earth (Hartmann and Davis 1975; Cameron and Ward 1976; Canup and Asphaug 2001; Canup 2004). In this scenario, formation of the inner planets require collisional accumulation of seed bodies, named 'planetoids' by Hills (1973) and the Moon formed by accumulation of the debris ejected after impact of a large differentiated object at the terminal stage of the formation of the Earth. We investigate here the probability of the capture of some of these 'planetoids' by the Earth in geocentric orbits and their probable role in modification of the primitive lunar crustal surface, e.g., formation of large mare basins. For this purpose, we proceed in the following manner.

We follow the approach of Hills (1973) and first calculate the most probable radius of "planetoids', which accumulate and form planetesimals or

Keywords. Planetary formation; planetesimals; planetoids; hybrid symplectic integrator; moonlets. 
planets. Then we use the hybrid symplectic integrator and simulate the formation of inner planets, calculating the probability of capture of planetoids in geocentric orbits during the accumulation process. These results are discussed and compared with other similar calculations and observations.

\subsection{Standard model of planet formation}

According to the standard model, the Sun formed as a result of gravitational instability in a dense interstellar molecular cloud. A small (2-10\%) portion of the gas and dust formed a flattened disk around the evolving Sun and contained most of the angular momentum of the system. This disk of dust and gas is termed as 'the solar nebula'. Computations fix the mass of the nebula in the range 0.01 to 0.07 solar masses. In this stage, the size of the grains was in the sub-micron range. These grains then aggregated and grew to centimeter and to meter size, initially by cohesion, primarily due to nongravitational forces like turbulence and gas drag, whereas gravitational attraction dominated subsequent formation of kilometer sized bodies. The time taken for formation of dust grains of $1 \mu \mathrm{m}$ is estimated to be $\sim 10^{5} \mathrm{yr}$ and for these particles to grow to $1 \mathrm{~cm}$ diameter, the time period of the order of decades is sufficient. The dust condensates (at $1 \mathrm{AU}$ ) evolved into solid bodies of $\sim 10 \mathrm{~km}$ diameter (planetoids) on time scales of the order of $\sim 10^{4} \mathrm{yr}$. In the next stage, these planetoids grew in to planetesimals $(\sim 1000 \mathrm{~km})$ while the disk is cleared of the dust. Some planetesimals serve as the embryos of the future planets. The time scale of the formation of planetesimals and planets is determined by their relative velocity, which, in turn, is determined by the mass distribution of the growing swarm and their mutual gravitational perturbations. Calculations of this stage of growth indicate that bodies as large as $10^{26} \mathrm{~g}(\sim 4000 \mathrm{~km})$ can form in $\sim 10^{5} \mathrm{yr}$. The present total mass of terrestrial planet region (Mercury to Asteroids) is $1.18 \times 10^{28} \mathrm{~g}$, of which $51 \%$ is in the Earth-Moon system. Therefore the growth of the Earth and terrestrial planets requires accumulation of a large number of planetesimals. The growth of planetesimals up to this stage can occur in two ways; (1) runaway accretion and (2) non-runaway accretion. Both these mechanisms converge on the time scale of formation of planets, i.e., $10^{8} \mathrm{yr}$, but the velocity and mass distributions obtained for the two processes are considerably different.

The final stage of growth of terrestrial planets is characterized by the emergence, after $\sim 10^{5} \mathrm{yr}$, of dominant embryos of the two large terrestrial planets, Earth and Venus, in orbits of relatively low eccentricity and inclination. As a result of gravitational perturbations associated with close encounters of the smaller $10^{26}-10^{27} \mathrm{~g}$ embryos with the Earth and Venus, the remainder of the material will probably be found in more eccentric orbits (e $\sim 0.1-0.3)$ that span the terrestrial planet region, eliminating local 'feeding zones' for each planet.

Final accumulation of Earth and Venus then includes giant impact events, i.e., collisions with the residual smaller planet size embryos. Such impacts have been associated with special events in terrestrial planet history, such as the formation of the Moon, the loss of Mercury's silicate mantle, retrograde rotation of Venus and the loss of Earth's original atmosphere. In these catastrophic impacts, much of energy would be deposited and distributed within the growing Earth, as found in numerical simulations of Benz et al $(1986,1987)$.

\subsection{Formation and evolution of the Moon}

The data collected by various missions to the Moon and the analysis of samples brought back by Apollo and Luna are consistent with the formation of the Moon by accumulation of the debris ejected as a result of a giant impact on the Earth (Hartmann 1986; Cameron 1986). Basically this hypothesis, called 'The Giant Impact Hypothesis' explains the similarity of isotopic composition of the Earth and Moon and deficiency of iron in the Moon (and its low density) compared to Earth. Subsequently, the Moon chemically differentiated to form a lowdensity highland crust and basaltic magma. The planetesimals continued to puncture the Moon's crust and formed the large basins, which were eventually filled with magma. These processes gave rise to features like MASCONS and hemispheric asymmetry.

Study of Mars, Mercury and the Moon's surfaces suggests that large scale cratering occurred on all the inner planets. Especially, surface of the Moon provides some evidence about the nature of impactors, which caused the vast basins. The data obtained by Ranger, Apollo and Clementine missions have given us some idea about the depth and diameter of some of the large basins (Williams and Zuber 1998; Wieczorek and Phillips 1999). The measured $d / D$ ratios are listed in table 1 . Some of these basins, particularly the front side mares, are partly filled with basalt to varying thickness and therefore it has not been possible to determine the depth of the various impact basins. An additional source of uncertainty is the viscous relaxation of the shape of the basins after they were formed. The data given in table 1 therefore underestimates the depth of the basins created by the impact, probably up to a factor of 2 . In comparison the observed extreme values of $d / D$ vary by a factor of 4 . These basins were created by impactors originating in the 
Table 1. Depth, diameter and approximate age of some selected basins (Williams and Zuber 1998).

\begin{tabular}{|c|c|c|c|c|}
\hline Basin & $\begin{array}{l}\text { Diameter } \\
(\mathrm{km})\end{array}$ & $\begin{array}{l}\text { Depth } \\
(\mathrm{km})\end{array}$ & $\begin{array}{l}\text { Error } \\
(\mathrm{km})\end{array}$ & Relative age \\
\hline Milne & 262 & 3.25 & 0.15 & Pre-Nectarian \\
\hline Bailly & 300 & 4.86 & 0.44 & Nectarian \\
\hline Schrodinger & 300 & 4.80 & 0.12 & Lower-Imbrian \\
\hline Planck & 320 & 4.00 & 0.29 & Pre-Nectarian \\
\hline Mendeleev & 325 & 4.98 & 0.16 & Nectarian \\
\hline Birkhoff & 330 & 4.76 & 0.19 & Pre-Nectarian \\
\hline Lorentz & 360 & 4.45 & 0.24 & Pre-Nectarian \\
\hline Korolev & 440 & 5.43 & 0.65 & Nectarian \\
\hline Moscoviensce & 445 & 5.25 & 0.50 & Nectarian \\
\hline Coulomb-Sarton & 530 & 4.50 & 0.20 & Pre-Nectarian \\
\hline Ingenii & 560 & 4.50 & 0.20 & Pre-Nectarian \\
\hline Hertzsprung & 570 & 5.31 & 0.77 & Nectarian \\
\hline Humboldtianum & 600 & 4.20 & 0.20 & Nectarian \\
\hline Freundlich-Sharonov & 600 & 6.00 & 1.12 & Pre-Nectarian \\
\hline Mendel-Rydberg & 630 & 5.24 & 0.87 & Nectarian \\
\hline South Pole-Aitken & 2500 & 8.53 & 0.53 & Pre-Nectarian \\
\hline
\end{tabular}

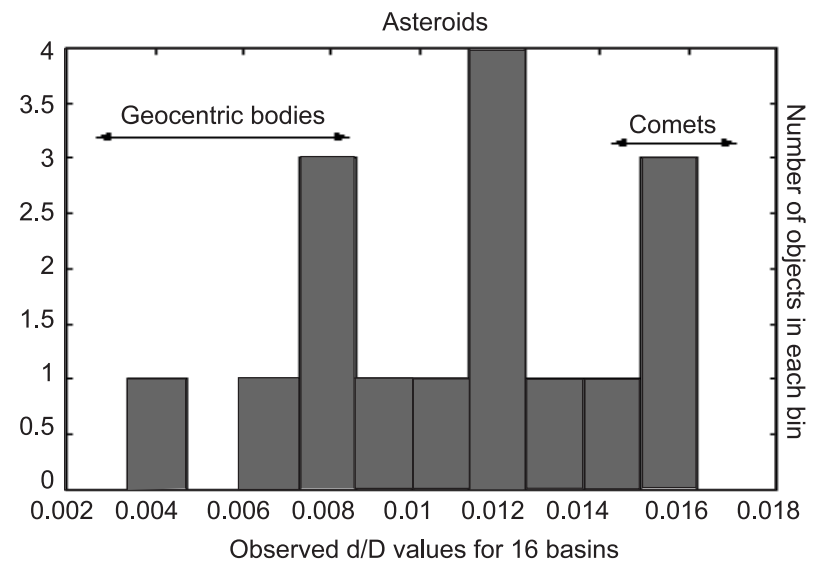

Figure 1. Depth/diameter distribution of 16 basins based on the data given by Williams and Zuber (1998).

interplanetary space like asteroids and comets. In addition, if there were some satellites of the Earth, formed together with the Earth or acquired by capture, they may have eventually fallen on the Moon or Earth, giving rise to impact basins.

If the depth to diameter $(d / D)$ ratio of impact basins depends primarily on velocity of impactor and, within a limited size range, is independent of its mass, three distinct peaks in $d / D$ distribution may be expected due to three different types of bodies, depending on their orbital characteristics, namely

- geocentric bodies are expected to have low velocity of impact,

- asteroids have higher velocity of impact and

- comets have the highest velocity of impact.

The data of table 1 are plotted in a histogram in figure 1. Although the data are limited, three distinct peaks can be seen in figure 1 .
It is interesting to ascertain if indeed the first peak in the distribution plot with smallest $d / D$ ratio (0.002 to 0.008$)$ is a result of the impact of geocentric bodies captured by the then forming Earth as 'Moonlets'. Hence we determine the probability of capture of planetesimals in geocentric orbits while the Earth was accumulating, following the procedure of Hills (1973) and Chambers (1999).

\section{Hills' approach}

Hills (1973) has calculated the number of bodies of various sizes present within the region of $2 \mathrm{AU}$ to few hundred AU while the planets were forming. He termed the bodies as 'planetoids', which he considers as the bodies from which the asteroids, comets and eventually planets have accumulated. We can consider these bodies as predecessors of planetesimals.

Modeling the process of accretion of individual asteroids, comets and planets formed by stochastic processes require a radius distribution function, specifically the total number of 'planetoids' and their most probable radius. Hills calculated the number of planetoids by a model of formation of seed bodies while their probable average radius was determined by the average pre-encounter velocity $(V)$ of the accretable material relative to an individual planetoid.

\subsection{The mean radius of planetoids}

Following Hills (1973), we assume $R$ as the radius of planetoid and mathematically allow $R \rightarrow \infty$, so that $N(\infty)$ is the total number of planetoids formed, then normalized integrated radius function 
is given in differential form by equation (11) of Hills (1973) as

$$
\begin{aligned}
d N(\ln R)= & (2 / \pi) \times N(\infty) \times\left(R / R_{c}\right) \\
& \times d\left[\ln \left(R / R_{c}\right)\right] /\left[1+\left(R / R_{c}\right)^{2}\right]
\end{aligned}
$$

where $R_{c}$ is the mean radius of the planetoid. This yields the value of $R_{c}=30 \mathrm{~km}$.

\subsection{Number of planetoids}

Assuming that $V$ was constant throughout the solar nebula, we can calculate the total number $N(\infty)$ of planetoids formed. The total mass of system of planetoids $M_{\text {total }}$, in which the largest body has a mass $M_{\max }$, is found by integrating (1a). This equation (equation (13) of Hills 1973) after integration and rearrangement of terms takes the form:

$$
N(\infty)=\frac{\pi \times\left(M_{\mathrm{total}} / M_{c}\right)}{\left(M_{\max } / M_{c}\right)^{2 / 3}-\ln \left[1+\left(M_{\max } / M_{c}\right)^{2 / 3}\right]} .
$$

Here $M_{c}$ is the critical mass corresponding to $R_{c}$.

Hills also calculated the influence of Jupiter's gravitational influence on the incoming comets up to $2 \mathrm{AU}$. Here we are interested in the situation around $1 \mathrm{AU}$, since planetoids in this region may be responsible for formation and evolution of the Earth.

We start at the 'planetoid' stage taking bodies with critical (mean) radius, and evolve them gravitationally to planetesimal stage and look closely at the environment around $1 \mathrm{AU}$.

For this purpose we take $n$-body approach using the hybrid symplectic integration technique given by Chambers (1999).

\section{Hybrid symplectic integrator}

For simulating the formation of planets by collisional accretion, we describe the equations of motion in the Hamiltonian sense, break the problem into a number of Hamiltonians and integrate each of these and finally add them up to find the velocity and position of each body in an approximate way. For this reason, the symplectic integration does not solve the problem exactly but gives an approximate solution within the prescribed limits of error. During the integration, when the bodies do not have any collisions, the equations of motion can be solved analytically using any conventional integrator to higher orders of accuracy, but when the bodies are on a collision course, it becomes difficult to solve the problem. If we divide the Hamiltonian of the system, $H$, into $H_{A}$ and $H_{B}$ where
$H_{A}$ takes care of gravitational interaction of every body with the central body (in this case the Sun) and $H_{B}$ takes care of gravitational interaction of all other bodies with the body under consideration except the Sun, then during an encounter between bodies $\alpha$ and $\beta$, their separation $r_{\alpha \beta}$ becomes small and makes one of the terms in $H_{B}$ comparable to $H_{A}$ and the error per step increases. This problem can be remedied if $H_{B}$ can be made small again compared to $H_{A}$ as is done using 'hybrid symplectic integrator'. In this approach, each of the interaction terms between $H_{A}$ and $H_{B}$ are split in such a way that the part in $H_{B}$ always remains small, while the part in $H_{A}$ is only evaluated during a close encounter in the following manner:

$$
\begin{aligned}
H_{A}= & \sum_{i=1}^{N}\left(\frac{p_{i}^{2}}{2 m_{i}}-\frac{G m_{\Theta} m_{i}}{r_{i \Theta}}\right) \\
& -G \sum_{i=1}^{N} \sum_{j=i+1}^{N} \frac{m_{i} m_{j}}{r_{i j}}\left[1-K\left(r_{i j}\right)\right], \\
H_{B}= & -G \sum_{i=1}^{N} \sum_{j=i+1}^{N} \frac{m_{i} m_{j}}{r_{i j}} K\left(r_{i j}\right),
\end{aligned}
$$

when $r_{i j}$ is large, $K$ tends to 1 , and when $r_{i j}$ is small, $K$ tends to zero. This ensures that $H_{B}$ is much less than $H_{A}$ and can be computed even during a close encounter. In the absence of an encounter, the terms in $H_{A}$ can be advanced analytically. In the above equation ' $p$ ' is the momentum term, $m_{j}$ is the mass of the Sun and $r_{i j}$ is the distance of ' $i$ th' body from the Sun. The change over function $K\left(r_{i j}\right)$ is determined by trial and error.

The advantages of using hybrid symplectic integrators are (i) no long term build up of energy error as the integrator solves the equations of motion and (ii) faster calculations for problems in which most of the mass is contained in a single body since the dominant force on each object can be 'built in' leaving only smaller perturbations to constrain the time step.

The code 'Mercury', kindly provided by $\mathrm{J}$ E Chambers, was a serial code requiring a long time for computation. Hence we parallelized the code using 'OpenMP' directives and used it for the simulation.

The inputs that were given to this code are as follows:

' $m$ ' is a real number, defines the body's mass in solar masses.

' $r$ ' is a real number, gives the maximum distance from the body (in Hill radii) that constitutes a close encounter.

' $d$ ' is a real number, gives the density of the body in $\mathrm{g} / \mathrm{cm}^{3}$. 
$a 1, a 2, a 3$ are real numbers, to indicate the nongravitational force parameters for any particular body, taken to be zero here.

The three initial coordinates and three velocity components are generated using Monte Carlo Technique, the conditions imposed being that any two bodies can not have the same distance from the central body but can have similar velocity. In addition, we use the three components of spin angular momentum for the body, in units of solar masses $\times \mathrm{AU}^{2}$ per day.

In this simulation, mass ' $m$ ', ' $r$ ' the Hills radius, density ' $d$ ' and the non-gravity force parameters are common along with the 3 spin angular momentum components and are taken to be $1.8 \times 10^{-13} \mathrm{~kg}, 1,3.1 \mathrm{~g} / \mathrm{cm}^{3}, 0,0,0$ and 0,0 , 0 respectively for the $30 \mathrm{~km}$ planetoids. The velocity ranged from 2 to $5 \mathrm{~km} / \mathrm{s}$ and the distribution of the bodies ranged from 1 to $50 \mathrm{AU}$. We also included the four inner planets in $50 \%$ formed condition and core of the Jupiter, in their respective current positions.

\section{Results and discussion}

The input was generated at the super computing facility at Physical Research Laboratory,

Table 2. Number of objects remaining at different time intervals in the simulation.

\begin{tabular}{ll}
\hline Time (starting at 4 million years) & Number of particles \\
\hline 0 & $\sim 1,500,000+5$ planets \\
10,000 years & $\sim 600,000+5$ planets \\
5 million years & $\sim 100,000+5$ planets \\
15 million years & $\sim 20,000+5$ planets \\
25 million years & $\sim 1,000+5$ planets \\
30 million years & 5 planets \\
50 million years & 5 planets \\
\hline
\end{tabular}

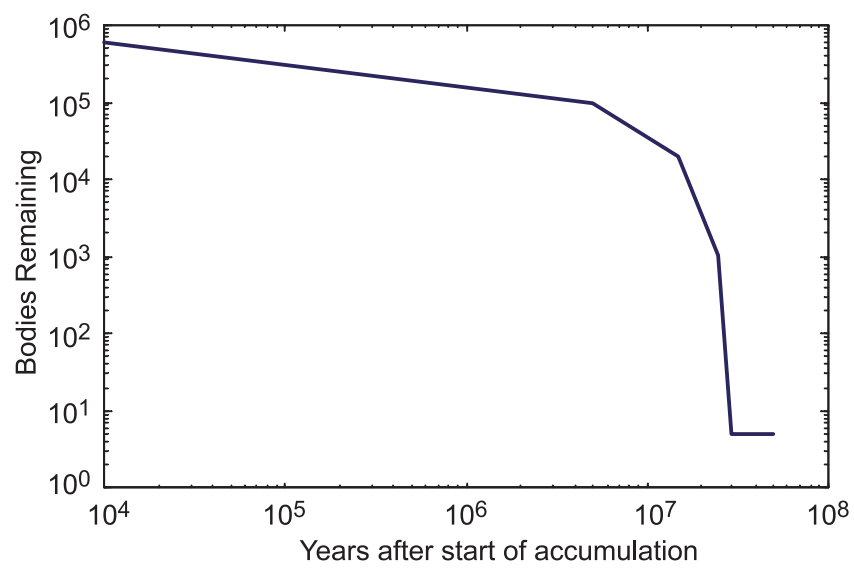

Figure 2. Number of bodies remaining in the interplanetary space as a function of time. The number of bodies decreases sharply as time progresses and finally only 5 planets remain.
Ahmedabad and the final simulation was carried out using the super computer at the Center for Modeling, Simulation and Design, University of Hyderabad, Hyderabad.

The program started with $\sim 1.5$ million objects and the 5 planets as described above, and has the provision of creating a dump list of the remaining objects at a specific interval of time (chosen as 500 years). This dump list was analyzed after the program got completely executed. Table 2 gives the number of bodies at some important stages, as seen in the dump list and is plotted in figure 2. Analysis of the file in which the ejected particles are dumped at various instances during the integration indicates that around $30-40 \%$ of the total 1.5 million particles of the considered bodies were ejected out.

From this simulation we infer that:

- Planetary accretion process in the terrestrial planet region seems to end by $\sim 30$ million years if we consider an input swarm of 1.5 million bodies (with an approximate total mass of $50 \%$ of these planets). This result is consistent with the work of Chambers and Wetherill (1998).

- The input mass should be about $40 \%$ more than the total planetary masses, to account for the ejected mass, for the planets to be fully formed.

- Negligible number of objects are captured within the roche lobe of the Earth in geocentric orbits while the Earth is being formed. Considering the errors of our computation, this number can be less than 5 .

These objects, in geocentric orbits, may eventually impact on the Earth or the Moon, resulting in a few of the old basins seen on the Moon. This is consistent with the observations in figure 1 .

Recent observations show that 'quasi Moons' like Asteroid 3753 Cruithne, Asteroid 2002 AA29 and Asteroid 2003 YN17 are present in temporary circumterrestrial orbits. Presently the density of interplanetary bodies is orders of magnitude smaller than that during the accumulation era. Based on these observations, during the formative epoch of the Earth, many more objects are expected to be captured in geocentric orbits. In this respect, the present simulation needs further modification. The simulation can be improved by including:

- various possibilities for an efficient capture and/or growth of Moonlets in Lagrangian points (e.g., L4 and L5) of the Sun-Earth system as Belbruno and Gott (2005) proposed or

- a set of tighter constraints on orbital parameters of planetoids, to efficiently deliver objects into geocentric orbits. 
If Moonlets in geocentric orbits were present when the Giant Impact took place on the Earth, then there is a possibility that the core of the Moon is made of such a Moonlet, which helped in quickly sweeping the debris ejected from the Earth. The seismic experiment on Japanese Lunar-A mission may be able to shed some light on the nature of the lunar core and confirm if the lunar core is made of a tellurian Moon.

If the number of Moonlets in geocentric orbits were large and they survived the formation of the Moon but eventually fell on Earth or the Moon, then we have to reconsider the role of the Late Heavy bombardment by interplanetary objects which is believed to be responsible for the creation of large lunar basins. It may, therefore, be useful to look at the isotopic, chemical and biological records of the Earth and the Moon to search for any evidence of this era.

\section{Terrestrial and lunar evidence for early large impacts}

The earliest records of life on Earth go back to 3.5 billion years and several attempts have been made to see if life appeared earlier. Since the old rocks from Isua in Greenland and Akilia, off the west coast of Greenland, are highly metamorphosed and the fossil records have been altered or destroyed, Arrhenius and colleagues (Mojzsis et al 1996; van Zuilen et al 2002), studied the carbon isotopic evidence which are characteristically biogenic and have shown that life probably originated on the Earth as early as 3.8 billion years ago, too close to the epoch of late heavy bombardment (Tera et al 1974; Wetherill 1975; Wasserburg et al 1977; Koeberl et al 2000; Arrhenius and Lepland 2000). Although this evidence has been disputed recently (Lepland et al 2005), there is a firm evidence of the presence of fossils at $3.5 \mathrm{~b}$.y. It is believed that a long time is required after a large catastrophic impact to restore conditions in which life can originate (Sleep et al 1989; Zahnle and Sleep 1997), and therefore this evidence is taken against late heavy bombardment of the Earth. These evidences can be reconciled if the Moon is subjected to heavy bombardment and the Earth is relatively spared of this intensely catastrophic and sterlising phase, preserving conditions congenial for life to originate. This can be accomplished by a large amount of big size debris, co-rotating with the newly formed Moon, which are preferentially swept by the receding Moon and do not much affect the Earth. Canup and Esposito (1996) have discussed various scenarios associated with the formation of the Moon with impact-generated disk including seeding with large intact fragments. The calculations presented here, have a bearing on the hypothesis of capture of a number of tellurian Moons around the Earth, which may be responsible for modification of the lunar surface by subsequent impacts and evolution of its surface. Further improvement in the computations is required to ascertain if indeed a number of Moonlets were present around the Earth at the time of the Giant impact.

\section{Acknowledgements}

We are grateful to $\mathrm{J}$ E Chambers for making his program 'Mercury' available to us for these computations. We thank the Center for Modeling, Simulation and Design, University of Hyderabad, for allowing us to use the Super Computer for some of the calculations reported here.

\section{References}

Arrhenius G and Lepland A 2000 Accretion of Moon and Earth and the emergence of life; Chemical Geology 169 69-82.

Belbruno E A and Gott J R 2005 Where did the Moon come from?, Astronom. J. 129 1724-1745.

Benz W, Slattery W L and Cameron A G W 1986 The origin of the Moon and the single impact hypothesis I; Icarus 66 515-535.

Benz W, Slattery W L and Cameron A G W 1987 The origin of the Moon and the single impact hypothesis II; Icarus $7130-45$

Cameron A G W 1986 The impact theory for Origin of the Moon; In: Origin of the Moon (eds) W K Hartmann, R J Phillips and G J Taylor (Houston: Lunar Planet. Institute) pp. 609-616.

Cameron A G W and Ward W R 1976 The origin of the Moon; (abstract) in Lunar Science VII, (Houston: Lunar Science Institute) pp. 120-122.

Canup R M 2004 Formation of the Moon; Ann. Rev. Astron. Astrophys. 42 441-475.

Canup R M and Esposito L W 1996 Accretion of the Moon from an Impact generated Disk; Icarus 119 427-446.

Canup R M and Asphaug E 2001 The Lunar-Forming Giant Impact; Nature 412 708-712.

Chambers J E 1999 A Hybrid Symplectic Integrator that Permits Close Encounters between Massive Bodies; Monthly Notices Royal Astronom. Soc. 304 793-799.

Chambers J E and Wetherill G W 1998 Making the terrestrial planets: N-body integrations of planetary embryos in three dimensions; Icarus 136 304-327.

Hartmann W K 1986 Moon Origin: the Impact trigger hypothesis; In: Origin of the Moon (eds) W K Hartmann, R J Phillips and G J Taylor (Houston: Lunar Planet. Institute) pp. 579-608.

Hartmann W K and Davis D R 1975 Satellite sized planetesimals and lunar origin; Icarus 24 504-515.

Hills J G 1973 On the process of Accretion in the formation of the Planets and Comets; Icarus 18 505-522.

Koeberl C, Reimold W U, McDonald I and Rosing M 2000 Search for petrographical and geochemical evidence for late heavy bombardment on Earth in Early Archaen rocks from Isua, Greenland; In: Impacts and the early Earth (eds) I Gilmour and C Koeberl, Lecture Notes in Earth Sciences 91 (Heidelberg: Springer Verlag) pp. 73-97. 
Lepland A, van Zuilen M A, Arrhenius G, Whitehouse M J and Fedo C M 2005 Questioning the evidence for Earth's earliest life-Akilia revisited; Geology 33 77-79.

Mojzsis S J, Arrhenius G, McKeegan K D, Harrison T M, Nutman A P and Friend R L 1996 Evidence for life on Earth before 3800 million years ago; Nature $\mathbf{3 8 4}$ $55-59$.

Safronov V S 1969 Evolution of the Protoplanetary cloud and formation of the Earth and Planets; (Nauka, Moscow), Translated in 1972 as NASA TT F-677.

Sleep N H, Zahnle K J, Kasting J F and Morowitz H J 1989 Annihilation of ecosystems by large asteroid impacts on the early earth; Nature 342 139-142.

Tera F, Papanastassiou D A and Wasserburg G 1974 Isotopic evidence for a terminal lunar cataclysm, Earth Planet. Sci. Lett. 22 1-21.

van Zuilen M A, Lepland A and Arrhenius G 2002 Reassessing the evidence for the earliest traces of life; Nature $\mathbf{4 1 8}$ 627-630.

Wasserburg G J, Papanstassiou D A, Tera F, Huneke F 1977 Outline of a lunar chronology; Trans. Royal. Soc. London 285 7-22.
Wetherill G W 1980 Formation of the Terrestrial Planets; Ann. Rev. Astron. Astrophys. 18 77-113.

Wetherill G W 1986 Accumulating the terrestrial planets and implications concerning lunar origin; In: Origin of the Moon (eds) W K Hartmann, R J Phillips and G J Taylor (Houston: Lunar Planet. Institute) pp. 519-550.

Wetherill G W and Stewart G R 1993 Formation of planetary embryos: Effects of fragmentation, low relative velocity and independent variation of eccentricity and inclination; Icarus 106 190-209.

Wetherill G W 1975 Late heavy bombardment of the Moon and terrestrial planets; Proc. Lunar Sci. Conf. 6th, pp. 1539-1561.

Wieczorek M A and Phillips R J 1999 Lunar Multi ring basins and the cratering process; Icarus 139 246-259.

Williams K K and Zuber M T 1998 Measurement and Analysis of Lunar Basin Depths from Clementine Altimetry; Icarus 131 107-122.

Zahnle K J and Sleep N H 1997 Impacts and the early evolution of life; In: Comets and the origin and evolution of life (eds) P J Thomas, C F Chyba and C P Mc Kay (New York: Springer Verlag) pp. 175-208. 